\title{
PERFIL DA FAMÍLIA CUIDADORA DE IDOSO DOENTE/FRAGILIZADO DO CONTEXTO SOCIOCULTURAL DE FLORIANÓPOLIS, SC ${ }^{1}$ PROFILE OF THE FAMILY CAREGIVER FOR FRAIL/SICK ELDERLY IN THE SOCIOCULTURAL CONTEXT OF FLORIANÓPOLIS, SC EL PERFIL DE LA FAMILIA CUIDADORA DEL ANCIANO ENFERMO EN EL CONTEXTO SOCIOCULTURAL DE FLORIANÓPOLIS-SC
}

\author{
Lucia Hisako Takase Gonçalves², Angela Maria Alvarę², Edite Lago da Silva Sena ${ }^{4}$, Luzia Wilma da Silva Santana4, \\ Fernanda Regina Vicente ${ }^{5}$
}

\begin{abstract}
${ }^{1}$ Parte da pesquisa originada do Convênio Coordenação de Aperfeiçoamento de Pessoal de Nível Superior (CAPES)/Programa de Qualificação Interinstitucional (PQI): Universidade Federal de Santa Catarina (UFSC)/Programa de Pós-Graduação em Enfermagem (PEN) e Universidade Estadual do Sudeste da Bahia (UESB)/Departamento de Saúde (DS) (2003-2007).

${ }^{2}$ Doutora em Ciências da Enfermagem. Professora Adjunto do Departamento de Enfermagem da UFSC. Líder do Grupo de Estudos sobre Cuidados de Saúde de Pessoas Idosas (GESPI).

${ }^{3}$ Doutora em Filosofia da Enfermagem. Prof. Adjunto do Departamento de Enfermagem da UFSC. Líder do GESPI.

${ }^{4}$ Doutoranda no Programa de Pós-Graduação de Enfermagem da UFSC. Participante do Convênio CAPES/PQI. Docente de Enfermagem da UESB/DS.

${ }^{5}$ Aluna de Enfermagem da UFSC. Bolsista Iniciação Científica (CNPq), Proc. 502506/2004-0.
\end{abstract}

PALAVRAS-CHAVE: Idoso. Cuidadores. Família.

KEYWORDS: Aged. Caregivers. Family.

PALABRASCLAVE: Anciano. Cuidadores. Familia.
RESUMO: Pesquisa avaliativo-diagnóstica com objetivo de traçar o perfil da família cuidadora de idoso. Foi desenvolvida aplicando-se os questionários: QPFC e WHOQOL-Breve, durante o ano de 2005. Amostra de 115 cuidadores foram selecionados entre os idosos cadastrados nos PSFs de Florianópolis. Resultados: os cuidadores, a maioria representada por mulher, atuavam em grande parte como cuidadoras únicas em tempo prolongado. Elas referiam dificuldades econômicas por terem de deixar o trabalho; sofriam de doenças como: HA, DM, osteomusculares. Contudo, as cuidadoras não se aborreciam em cuidar do idoso face os comportamentos repetitivos ou cuidados de difícil realização. Atribuíam-se como cuidadoras por aceitar a missão necessária e dignificante, como parte dos princípios morais e religiosos. Em conclusão, os familiares cuidadores de idosos doentes e/ou fragilizados, continuam sendo preponderantemente mulheres, inclusive idosas. Tal resultado impõe aos Programas de Saúde da Família de idosos em condição de dependência, atenção específica, que contemple o binômio idoso/cuidador.

ABSTRACT: The objective of this evaluative-diagnostic research was to draw the profile of the elderly caregiver family and was developed through the application of the QPFC and WHOQOL-brief questionnaires in 2005. The sample was composed of 115 caregivers selected among elderly caregivers registered in the Family Health Programs (PSFs) of Florianópolis, SC, Brazil. Results: the caregivers, mostly women, acted as the sole caregiver during extended periods of time. They reported economical difficulties because they had to leave their jobs and presented diseases such as: HA, MD, and Osseo-muscular diseases. However, they did not tire of caring for the elderly in relation to repetitive tasks or care that is difficult to perform. Instead, they were resigned and accepted the necessary and dignifying mission as part of their moral and religious principles. Conclusion: although they seem to be qualified, caregivers perform functions in not very favorable conditions, which obligate public health services investment to support them.

RESUMEN: Investigación evaluativa-diagnóstica con el objetivo de trazar el perfil de la familia cuidadora del anciano. Se desarrollo aplicándose los cuestionários: QPFC y el WHOQOL-Breve, durante el año del 2005. La muestra de 115 cuidadores fueron selecionados entre los ancianos registrados en el PSFs de Florianópolis. Los resultados indican que los cuidadores en su mayoría eran mujeres. Ellas actuaban como cuidadoras únicas a tiempo prolongado y atravesaban dificuldades económicas por tener de abandonar el trabajo. Las cuidadoras no se incomodaban con la tarea del cuidado frente a los comportamientos repetitivos o con cuidados complejos. Considerábanse como cuidadoras por aceptar la misión como necesaria y digna de los principios morales y religiosos. Se concluye que las cuidadoras realizan funciones en condiciones poco favorables, aunque se muestren vocacionadas para el cuidado, el cual impone a los Programas de la Salud de la Familia una atención más específica que considere al binomio anciano/cuidador.
Endereço: Lucia Hisako Takase Gonçalves

R. Agostinho Sielski, 84

88.035-320 - Santa Mônica, Florianópolis, SC.

E-mail: lucia@nfr.ufsc.br; gespi@nfr.ufsc.br
Artigo original: Pesquisa

Recebido em: 15 de maio de 2006.

Aprovação final: 17 de outubro de 2006. 


\section{INTRODUÇÃO}

$\mathrm{O}$ artigo apresenta parte do relatório de pesquisa de natureza multicêntrica realizada na cidade de Florianópolis, SC, sob os auspícios de um convênio interinstitucional, cujo objetivo central foi conhecer o perfil da família cuidadora de idoso doente e/ou fragilizado, convivendo em diferentes contextos socioculturais. ${ }^{1}$

Embora seja ainda um país jovem, o Brasil vem demonstrando um perfil populacional do tipo de transição demográfica que sinaliza rápida mudança em termos de aumento vertiginoso do estrato idoso da população, representado no ano de 2000 por $8,6 \%$ do total, segundo Instituto Brasileiro de Geografia e Estatística (IBGE), mas com projeções estatísticas apontando para 15\% em 2025. Aumento esse que representa, do ponto de vista da adoção de políticas públicas e sociais, solução de difícil alcance para contemplar adequadamente as peculiaridades emergentes dessa população idosa, caso a questão não seja tomada a sério com antecipação.

Integrando o contingente de pessoas idosas evidencia-se o fenômeno do prolongamento da vida, pois viver até os 80 anos de idade, nas últimas décadas, já não é algo surpreendente. ${ }^{2}$ Contudo, a condição de longevidade associa-se a fragilização pelo envelhecimento, tornando o idoso vulnerável às diversas situações de vida e saúde. No contexto brasileiro estima-se que $85 \%$ dos idosos apresentem pelo menos uma doença crônica, e destes pelo menos 10\% com sobreposição de afecções concomitantes. Desse modo, a situação de cronicidade e longevidade atual dos brasileiros contribuem para o aumento de idosos com limitações funcionais, implicando em necessidade de cuidados constantes.

Geralmente esses cuidados são prestados pela família e pela comunidade, sendo o domicílio o espaço sociocultural natural. No que se refere à família, o cuidado normalmente incide em um de seus membros, o qual é denominado cuidador principal por ser o responsável pelos cuidados do idoso. Outros membros da família podem auxiliar em atividades complementares, daí serem chamados de cuidadores secundários. ${ }^{3-5}$

Diversos motivos contribuem para que uma pessoa se torne cuidadora principal, dentre os quais se destacam: a obrigação moral alicerçada em aspectos culturais e religiosos; a condição de conjugalidade, o fato de ser esposo ou esposa; a ausência de outras pessoas para a tarefa do cuidar, caso em que o cuidador assume essa incumbência não por opção, mas, na maioria das vezes, por força das circunstâncias; as dificuldades financeiras como em caso de filhas desempregadas que cuidam dos pais em troca do sustento. ${ }^{5}$

$\mathrm{Na}$ maioria dos países, observa-se que ao longo da história o cuidado do idoso é exercido por mulheres. Também em nosso meio as cuidadoras são, principalmente, as esposas, as filhas e as netas. Tal fato pode ser explicado pela tradição de no passado recente as mulheres não desempenharem funções fora de casa, justificando sua maior disponibilidade para o cuidado da família. Contudo, essa realidade vem sendo modificada em função da inserção social da mulher participando progressivamente no mercado de trabalho. Os estudos em nosso meio apontam que, geralmente, as cuidadoras residem com o idoso, são casadas e, por isso, somam às suas atividades de cuidar as atividades domésticas próprias de mãe, esposa e avó, gerando um acúmulo de trabalho em casa e uma sobrecarga nos diversos domínios da vida da cuidadora, como: social, físico, emocional, espiritual, enfim, contribuindo para o autodescuido da própria saúde. ${ }^{5-8}$

Um dos aspectos que afetam o cotidiano da maioria das famílias cuidadoras é a dificuldade financeira da camada mais pobre da população. Muitos cuidadores estão desempregados e sobrevivem dos recursos provenientes da aposentadoria do idoso que, em muitos casos, são insuficientes para atender as necessidades básicas do próprio idoso. ${ }^{2}$

Outros estudos apontam a interferência de forma significativa no processo de cuidar de idosos, especialmente nos casos de portadores de demência, os quais necessitam de cuidados especiais e expõem o cuidador a estresse prolongado. Nesses casos, além de treinamento específico para lidarem com a situação de cuidar de outrem, os cuidadores precisam de suporte social para ajudá-los a manter a própria saúde e poder cuidar de si mesmos. Não dispondo de tal suporte, os cuidadores ficam expostos a riscos de adoecer, não pelo cuidado em si, mas pela sobrecarga a que são impostos., ${ }^{3,7}$

Embora a literatura atual sinalize as múltiplas características do familiar cuidador de idosos no âmbito domiciliar, há ainda necessidade de explorar mais conhecimentos, considerando tais características tomarem contornos diversos segundo as especificidades regionais do país, motivo porque foi realizada a presente pesquisa. 


\section{METODOLOGIA}

Pesquisa de natureza multicêntrica, do tipo exploratório-descritivo, teve o propósito diagnóstico-avaliativo para traçar o perfil do familiar cuidador principal do idoso doente e/ou fragilizado, vivendo na comunidade em contexto domiciliar. $\mathrm{O}$ familiar cuidador, sujeito desta pesquisa, era aquele que cuidava do idoso portador de quaisquer doenças crônicas, geralmente sofrendo de comorbidade ou fragilizado em função da situação de cronicidade e do próprio avanço da idade levando-o a um estado de incapacidade e dependência de outrem para os cuidados nas atividades da vida diária. Buscaram-se os dados para o desenho do referido perfil por meio da aplicação do Questionário de Perfil da Família Cuidadora (QPFC). Tal questionário, subdividido em três partes, incluía na primeira: a identificação do familiar cuidador principal, destacando as variáveis sociodemográficas, o estado de saúde e a qualidade de vida; na segunda: as características do estado de saúde do idoso em cuidado e necessidades envolvidas; e na terceira, identificação do contexto da relação do cuidador com a pessoa idosa cuidada. Para avaliar a qualidade de vida do cuidador foi adicionada a aplicação do WHOQOL-Breve (Questionário de qualidade de vida da OMS). ${ }^{9}$

O QPFC foi elaborado pela equipe de pesquisa do Grupo de Estudos de Cuidados de Saúde de Pessoas Idosas (GESPI) ${ }^{10}$ e submetido a testes em diferentes contextos de aplicação. Sua construção teve base em diversas obras nacionais recentes, ${ }^{2,4,5}$ além da experiência da própria equipe e, especialmente, na Encuesta a Personas Cuidadoras aplicada em âmbito nacional da Espanha pelo Centro de Investigaciones Sociológicas (CIS) do Instituto de Migraciones y Servicios Sociales (IMSERSO) do Ministério do Trabalho e Assuntos Sociais da Espanha. ${ }^{11}$

A amostra, do tipo intencional, foi constituída de 115 familiares cuidadores selecionados de uma população identificada nas unidades básicas de saúde com Programa de Saúde da Família (PSF) do município de Florianópolis, SC no período compreendido entre 2004 e 2005. Florianópolis tem uma população idosa correspondente a $8,4 \%$ da população total, o que em números absolutos significa 28.843 habitantes idosos segundo o Instituto Brasileiro de Geografia e Estatística (IBGE).

Em sua concepção geral para aplicação em multicentros, o projeto foi encaminhado ao Comitê de Ética em Pesquisa com Seres Humanos da UFSC e devidamente protocolado, analisado e aprovado, segundo as exigências da Resolução 196/96 do CNS do MS.

\section{RESULTADOS E DISCUSSÃO}

\section{Cuidadores: caracterização, estado de saúde e qualidade de vida}

Dos 115 familiares cuidadores pesquisados, a grande maioria representou o gênero feminino $(84,3 \%)$, e a média de idade apresentada foi de 48,5 anos $(58,2 \%)$. Tais características se mostram semelhantes as das pesquisas nacionais mais destacadas e também do perfil de cuidadores de idosos do CIS/IMSERSO da Espanha. ${ }^{11}$ Os dados continuam demonstrando que os cuidadores de idosos são predominantemente mulheres de meia idade, contudo, convém destacar a crescente participação dos homens em diferentes idades, a exemplo deste estudo, no qual aparecem $15,7 \%$ de esposos, filhos e netos.

Observou-se ainda que 58,2\% dos cuidadores eram casados, $37,3 \%$ separados ou solteiros, e 3,4\% viúvos. Em relação à escolaridade, 50,3\% cursaram somente o ensino básico; $32,1 \%$ ensino médio; $15,6 \%$ ensino superior e $1,7 \%$ referiram ser analfabetos. Quanto à ocupação 53\% referiram não ter atividades extradomiciliares, dedicando-se ao cuidado do idoso e de outros membros da família, além dos afazeres domésticos. Enquanto, os demais $47 \%$ conciliavam atividades de trabalho fora do lar com o cuidado do idoso. Assim como em algumas pesquisas citadas neste artigo também muitas cuidadoras foram instadas a deixar o trabalho ou reduzir sua jornada para cuidar do idoso, que em geral exigia dedicação permanente.

Questionados sobre sua situação de saúde, doenças ou problemas que os afetavam, os cuidadores referiram sofrer de hipertensão arterial e outros problemas cardiovasculares (16,5\%), seguido de problemas osteomusculares $(9,5 \%)$ e diabetes mellitus $(6,9 \%)$. Contudo, quando perguntados quanto à percepção de seu estado de saúde atual apenas $33,9 \%$ consideram como bom, $31,3 \%$ como regular e 4,3\% ruim. Quando solicitados a comparar seu estado de saúde ao de cinco anos atrás, 37,3\% disseram ter piorado.

Quanto à qualidade de vida, conforme Tabela 1 , a maioria $(69,5 \%)$ mostrou-se satisfeita. Tal constatação se deve possivelmente aos outros domínios inclusos na avaliação, como: físico, psicológico, relações sociais e meio ambiente. 
Tabela 1 - Distribuição das respostas do cuidador segundo os escores de qualidade de vida (WHOQOL-Breve) e gênero, no contexto sociocultural de Florianópolis, 2005.

\begin{tabular}{|c|c|c|c|c|c|c|c|c|c|c|c|c|}
\hline \multirow{3}{*}{ Gênero } & \multicolumn{12}{|c|}{ Qualidade de Vida } \\
\hline & \multicolumn{2}{|c|}{$\begin{array}{c}\text { Muito } \\
\text { Insatisfeito }\end{array}$} & \multicolumn{2}{|c|}{ Insatisfeito } & \multicolumn{2}{|c|}{ Regular } & \multicolumn{2}{|c|}{ Satisfeito } & \multicolumn{2}{|c|}{$\begin{array}{c}\text { Muito } \\
\text { Satisfeito }\end{array}$} & \multicolumn{2}{|c|}{ Total } \\
\hline & $n$ & $\%$ & $\mathbf{n}$ & $\%$ & $\mathbf{n}$ & $\%$ & $\mathbf{n}$ & $\%$ & $\mathbf{n}$ & $\%$ & $\mathbf{n}$ & $\%$ \\
\hline Feminino & 00 & 0.0 & 02 & 1.7 & 18 & 15.6 & 68 & 59.1 & 09 & 7.8 & 97 & 84.3 \\
\hline Masculino & 00 & 0.0 & 00 & 0.0 & 02 & 1.7 & 12 & 10.4 & 04 & 3.4 & 18 & 15.6 \\
\hline Total & 0 & 0.0 & 2 & 1.7 & 20 & 17.3 & 80 & 69.5 & 13 & 11.2 & 115 & 100 \\
\hline
\end{tabular}

Nota: Percentagem calculada sobre $100 \%$ da amostra de 115 sujeitos.

Familiares cuidadores: situação do cuidado

Quanto à dispensação do cuidado, o estudo revelou que $64,6 \%$ dos cuidadores atuavam de modo permanente, ou seja, dedicavam-se diuturnamente à atenção do idoso, investindo diariamente cinco horas ou mais em atividades de cuidado direto, como: ajuda no deslocamento, auxílio no banho, encaminhamento ao sanitário para as necessidades fisiológicas e em situações de incontinência urinária, ajuda no despirse e vestir-se e na troca de fraldas e roupas.

Semelhante a outros estudos sobre cuidador, este revelou também que o processo de cuidar do idoso em contexto domiciliar pode desencadear o aparecimento de limitações na vida cotidiana do cuidador com conseqüente risco à sua saúde e bemestar, o que pode ser observado na Tabela 2.

Tabela 2 - Freqüência de conseqüências advindas da tarefa de cuidar, segundo os cuidadores de idosos, Florianópolis, 2005.

\begin{tabular}{lcc} 
Conseqüências & \multicolumn{2}{c}{ Freqüência } \\
& $\mathbf{n}$ & \% \\
\hline Redução do tempo de lazer & 50 & 43.1 \\
Encontrar-se sempre cansada (o) & 39 & 33.6 \\
Não ter mais tempos para gastar com os amigos & 31 & 26.7 \\
Não poder sair de férias & 28 & 24.1 \\
Sentir a saúde piorada & 26 & 22.4 \\
Não poder trabalhar fora & 24 & 20.6 \\
Não ter tempo para cuidar-se & 25 & 22.5 \\
Sentir-se deprimida (o) & 23 & 19.9 \\
Aumento dos problemas econômicos & 21 & 18.1 \\
Ter conflitos com o cônjuge & 12 & 10.3 \\
Tive que deixar meu trabalho & 08 & 6.8 \\
Tive que reduzir minha jornada de trabalho & 08 & 6.8 \\
\hline
\end{tabular}

Nota: Percentagem calculada sobre $100 \%$ da amostra de 115 sujeitos.

O número significativo de cuidadores que se dedicam de modo permanente $(56,3 \%)$ revelado neste estudo ratifica dados de pesquisas anteriores. Cuidar de um idoso em tempo prolongado exige exposição constante dos cuidadores a riscos de adoecimento, pois principalmente aqueles que são cuidadores únicos assumem total responsabilidade, e com isso estão sempre sobrecarregados. Em se tratando de mulheres, estas acumulam diversos papéis como: de mãe, esposa e cuidadora de outros dependentes, dentre outros. Tal sobrecarga compromete o autodescuido, como se constata na resposta de 22,5\% (Tabela 2) dos cuidadores dizendo não ter mais tempo para cuidar de si próprios. Pode-se observar ainda que $43,1 \%$ dos cuidadores tiveram que reduzir seu tempo de lazer, 33,6\% encontravam-se sempre cansados, $24,1 \%$ não podiam sair de férias e $22,4 \%$ sentiam sua saúde piorada. 
Entre os cuidadores com dedicação permanente $(56,3 \%)$, estes têm outros dependentes além do idoso, como filhos $(38,2 \%)$, netos $(10,4 \%)$, cônjuge $(4,3 \%)$ e outros $(3,4 \%)$.

Os idosos cuidados por esses familiares cuidadores sofriam de doenças como: hipertensão arterial $(75,6 \%)$, diabetes mellitus (53\%), problemas cardiovasculares (49,5\%) e conseqüências de AVC (29,5\%), muitas vezes em situações de comorbidade.

Diante dessa diversidade de doenças crônicas e em circunstâncias de vida familiar das mais diversas, não bastam a disposição e a solidariedade dos cuidadores em assumir o cuidado do idoso, é fundamental que haja apoio institucional e orientações básicas de cuidado conforme as especificidades de cada situação.

O estudo mostrou ainda que $56 \%$ dos cuidadores têm parentes que ajudam no cuidado do idoso, porém, convém ressaltar que $28,4 \%$ dos cuidadores não recebem ajuda nenhuma, são cuidadores únicos.
A convivência intergeracional tem-se mostrado positiva à medida que membros da família assumem o papel de cuidadores secundários, minimizando situação geradora de estresse sobre a cuidadora principal, especialmente quando tem atuação permanente.

Com tais perspectivas, o conhecimento do perfil dos cuidadores e dos idosos cuidados por eles é essencial para subsidiar os serviços de saúde no planejamento e implementação da capacitação de cuidadores familiares, considerando-os também como clientes usuários que necessitam de atenção de saúde.

\section{Familiar cuidador: relação com o idoso}

Com relação aos comportamentos do idoso e cuidados do idoso nas atividades da vida diária (AVDs) como circunstâncias que podem ou não trazer constrangimentos, tal fato foi perguntado como sendo incômodo ou aborrecimento e, as respostas estão representadas nas Tabelas 3 e 4 .

Tabela 3 - Distribuição da presença de comportamentos comuns do idoso e respectivas respostas ao incômodo produzido nos cuidadores do contexto sociocultural de Florianópolis, 2005.

\begin{tabular}{lcccc}
\hline \multirow{2}{*}{ Comportamento do idoso } & \multicolumn{2}{c}{ Frequência } & \multicolumn{2}{c}{ Incômodo } \\
& $\mathbf{n}$ & $\%$ & $\mathbf{n}$ & $\%$ \\
\hline Só reclama da situação & 61 & 52.5 & 26 & 22.3 \\
Junta coisas inúteis & 54 & 46.5 & 20 & 17.3 \\
Tem alterações de sono & 63 & 54.3 & 19 & 16.3 \\
É agressivo verbalmente & 32 & 27.5 & 17 & 14.5 \\
Come coisas que não deve & 31 & 26.7 & 16 & 13.6 \\
Não gosta de tomar banho & 34 & 23.3 & 15 & 12.8 \\
Não se importa com a limpeza & 33 & 28.4 & 12 & 10.3 \\
Repete continuamente as mesmas histórias & 70 & 60.3 & 12 & 10.3 \\
Suja as roupas com urina e fezes & 40 & 34.4 & 10 & 8.6 \\
Necessita de troca de fraldas, no caso de incontinência urinária & 63 & 54.3 & 10 & 8.6 \\
\hline
\end{tabular}

Nota: Percentagem calculada sobre os $100 \%$ da amostra de 115 cuidadores.

Conforme se observa na Tabela 3 , os comportamentos comuns apresentados pelos idosos, sejam eles repetitivos de necessidades ou de queixas contínuas, em freqüência relativamente alta, houve em contraposição, respostas de freqüência relativamente baixa dos cuidadores, demonstrando que não se incomodavam nem se aborreciam com tais comportamentos dos idosos cuidados.

Embora aqui pareça que os cuidadores estejam afeitos ao cuidado dos idosos porque não se incomodam nem se aborrecem com os seus comportamentos, vale ressaltar questionamentos em alguns quesitos. Por exemplo: com relação ao comporta- mento do idoso de não gostar de higienização, até onde o não aborrecer-se por parte dos cuidadores significa: conivência com o idoso negligenciando o cuidado; ou, real paciência necessária e afetuosa de convencimento junto ao mesmo para os efetivos cuidados higiênicos necessários? Tal questão exige exploração de natureza qualitativa para captar as nuanças no convívio do processo de cuidar. ${ }^{8}$

Conforme se verifica na Tabela 4 , seguem os cuidados nas atividades da vida diária dos idosos que requeriam ajuda ou dispensa de cuidados pelos cuidadores. 
Tabela 4 - Distribuição das atividades da vida diária do idoso, as quais necessitam de ajuda do cuidador e suas respectivas reações de incômodo, do contexto sociocultural de Florianópolis, 2005.

\begin{tabular}{lcccc}
\hline \multirow{2}{*}{ Ajuda nas atividades da vida diária } & \multicolumn{2}{c}{ Frequência } & \multicolumn{2}{c}{ Incômodo } \\
& $\mathbf{n}$ & $\mathbf{\%}$ & $\mathbf{n}$ & \% \\
\hline Para alimentar-se & 37 & 31.8 & 11 & 9.3 \\
Para tomar banho & 58 & 49.9 & 10 & 8.5 \\
Trocar as fraldas no caso de incontinência urinária & 30 & 25.7 & 09 & 7.7 \\
Para chegar ao banheiro a tempo & 30 & 25.7 & 08 & 6.8 \\
Na administração da própria vida & 94 & 80.9 & 07 & 5.9 \\
Para locomoção/deslocamento & 61 & 52.5 & 05 & 4.2 \\
Para cuidar de suas finanças & 98 & 84.4 & 05 & 4.2 \\
Para sair para fazer comprar & 91 & 78.3 & 04 & 3.4 \\
\hline Nor Pera
\end{tabular}

Nota: Percentagem calculada sobre os $100 \%$ da amostra de 115 cuidadores.

Apesar de serem freqüentes a necessidade de ajuda ou dispensação de cuidados pessoais diretos e íntimos aos idosos, as respostas dos cuidadores de não se incomodarem nem se aborrecerem em desenvolver tais cuidados deixa parecer que esses cuidadores são vocacionados para a tarefa do cuidar do idoso. Contudo, merece conjecturas: o não se incomodar em ajudar a banhar o idoso, levar o idoso periodicamente ao sanitário, por exemplo, não poderia se constituir em atitude de negligência do cuidador quando este se dispõe a desenvolver tal cuidado somente quando lhe aprouver? Ou, não se incomodar em ajudar o idoso em deslocamentos apanhando uma condução, por exemplo, para levar à consulta médica ou de reabilitação: não poderia traduzir em uma inadequação no controle da saúde do idoso ao executar a tarefa somente quando houver recursos externos sem mobilização própria pessoal para tal requerimento? Tais conjecturas merecem exame detido da complexa dinâmica familiar no aspecto do processo de cuidar entre seus membros em meio a situação generalizada de precários recursos de atendimento nos serviços de saúde.

Acerca dos sentimentos de identidade do cuidador diante das atividades cuidativas junto ao idoso, os dados da Tabela 5 revelam que a maioria percebe o cuidado como algo que o dignifica como pessoa ou como cumprimento de um dever moral e de princípios religiosos, satisfação pela manifestação de gratidão pelo idoso, reconhecimento da família e da comunidade, embora seja pertinente destacar que alguns cuidadores são levados a assumir este papel por ser a única opção disponível.

Tabela 5 - Distribuição das afirmações com as quais os cuidadores se identificam em relação às tarefas desempenhadas no cuidado do idoso, Florianópolis, 2005.

\begin{tabular}{lcc}
\hline Motivos para ser cuidador & $\mathbf{n}$ & $\mathbf{\%}$ \\
\hline É algo que me dignifica como pessoa & 63 & 53.3 \\
Eu considero uma obrigação moral & 61 & 52.5 \\
A pessoa cuidada é muito agradecida & 53 & 45.6 \\
Minha família apóia e valoriza esse meu papel & 35 & 30.1 \\
Apoio-me em minhas convicções religiosas & 33 & 28.4 \\
O círculo de relações sociais valoriza os cuidados que presto ao idoso & 18 & 15.5 \\
É uma carga excessiva & 13 & 11.2 \\
Não há outro remédio se não cuidar & 09 & 7.7 \\
Pessoas da minha roda acham que eu não deveria fazer esse sacrifício & 08 & 6.8
\end{tabular}

Nota: Percentagem calculada sobre os $100 \%$ da amostra de 115 cuidadores. 
Tal constatação inicial está a merecer estudos de exploração mais aprofundada, de vez que não tem sido comum nos estudos, com ressalva à pesquisa cultural, na qual foi ressaltada a valorização da mulher cuidadora pela comunidade de antecedentes culturais italianos, num contexto interiorano brasileiro. ${ }^{12}$ Há também a pesquisa, cujo objetivo foi descrever a avaliação cognitiva (positiva e negativa) das informações dos cuidadores familiares de idosos dependentes, destacando entre os resultados os benefícios psicossociais do cuidar. ${ }^{13}$

Não obstante os estudos, tanto internacionais quanto nacionais continuarem mostrando que o cuidado ao idoso interfere de modo sombrio no viver do cuidador, resultando em sobrecarga e conseqüente estresse, este estudo faz aparecer uma faceta positiva, devendo esta ser estimulada, o que poderá contribuir para melhoria da auto-estima, e, conseqüentemente para mais saúde e bem-estar do cuidador.

Tornar-se cuidador(a) de um familiar idoso doente e/ou fragilizado, e com significativo grau de dependência não pertence à ordem do saber, mas constitui uma experiência ambígua e impessoal. Por mais que se tente encontrar motivos, a explicação sempre será incompleta. A reflexão nunca pode fazer com que deixemos de pensar com os instrumentos culturais preparados por nossa educação, nossos esforços precedentes, enfim, nossa história. ${ }^{14}$ Vale dizer: diante da necessidade de cuidar do outro, a pessoa é conduzida por uma experiência coexistencial ligados aos vínculos e às marcas afetivas ou não afetivas com aquele a quem se dirige o cuidado. É uma experiência caracterizada por uma espécie de não saber de si, a qual é captada pelo sensível. Nessa perspectiva, "cuidar é mais que um ato, é uma atitude. Portanto, abrange mais que um momento de atenção, de zelo e de desvelo. Representa uma atitude de ocupação, preocupação, de responsabilização e de envolvimento afetivo com o outro". ${ }^{15: 33}$

Sob esse olhar, a decisão de cuidar de um familiar idoso exprime o que há de mais essencial no humano que é aquilo que se diz dele, o Ser, e, em tal sentido, o cuidado é ontológico, ele é o próprio Ser. Esse olhar permite compreender a resposta de muitos cuidadores da cidade de Florianópolis, onde as pessoas estabelecem e mantêm vínculos afetivos e de gratidão e, mesmo em circunstâncias tão complexas como as situações que envolvem o ter que cuidar de um familiar idoso seriamente doente, o cuidador demonstra certo nível de satisfação e bem-estar. Muitos desses cuidadores já se encontram na velhice, sofrem de alguma doença crônica, mas ainda assim, sentem-se gratificados. Como os dados do presente estudo de perfil, cujo enfoque é mais de levantamento de situação de cuidado, as respostas dos cuidadores merecem outros estudos mais aprofundados, a fim de se obter não só uma explicação dos motivos por que os cuidadores de Florianópolis sentem-se gratificados e com certa satisfação, mas para compreender mais ampla e claramente como esses sentimentos se mostram para eles.

\section{CONCLUSÃO E IMPLICAÇÕES}

Os familiares cuidadores principais de idosos são primordialmente mulheres de meia idade, embora convenha destacar a participação de cuidadoras idosas cuidando de seus cônjuges idosos. Também merece menção a participação crescente de homens de diferentes idades, cuidando de idosos, contudo ainda é a mulher a grande parte deles cuidador único que além dessa função, tem sob seus cuidados familiares outros dependentes como: filhos(as), netos(as) e parentes portadores de cuidados especiais.

$\mathrm{Na}$ situação de cuidado prevalece o cuidar de modo permanente, de gasto diário de tempo com mais de 3 horas, e em cuidado prolongado por mais de três anos.

Entre as conseqüências da tarefa de cuidar do idoso, as cuidadoras tinham limitações na vida profissional, desde a redução da jornada de trabalho até o seu abandono. Em termos de queixa, as cuidadoras expressavam falta de tempo para se cuidar; convivência conjugal com conflitos; cansaço permanente e percepção de saúde piorada.

No processo de cuidar em geral, as cuidadoras não se aborreciam com os constrangedores comportamentos de idosos e cuidados pessoais mais complexos da vida diária, denotando adequação pessoal ao cuidado do idoso, constatação evidenciada nas respostas dadas de existência de relações afetivas normais, tanto antes como após o cuidado estabelecido junto ao idoso.

Entre os motivos atribuídos pelos cuidadores em assumir a responsabilidade pelo idoso, destacamse: "dignificação como pessoa”, "obrigação moral ou prática e princípios religiosos”, "reconhecimento do próprio idoso com manifestação de gratidão", "reconhecimento da família e da comunidade", e também como sendo a "única opção".

Face a diversidade de características reveladas pelos cuidadores do contexto sociocultural de Floria- 
nópolis, em comparação a outros contextos, ${ }^{1}$ tornase imprescindível a avaliação local e periódica, como o presente estudo, para subsidiar os serviços básicos de saúde de programas adequados voltados à família com membros idosos em situação de fragilização.

\section{REFERÊNCIAS}

1 Gonçalves LHT, Santos AS, Costa MA, Rossi CL. Profile of the family caregiver for a frail elderly relative in the domestic environment at the five sociocultural context of Florianópolis, Jequié, Passo Fundo, Rosario and Porto: abstract caderno do programa [CD-ROM]. In: Anais do 18o Congress of the International Association of Gerontology; 2005 Jun 26-30; Rio de Janeiro, Brasil. Rio de Janeiro: SBGG; 2005.

2 Camarano AA. Os novos idosos brasileiros muito além dos 60? Rio de Janeiro: IPEA; 2004.

3 Yuaso DL. Cuidar de cuidadores: resultados de um programa de treinamento realizado em domicílio. In: Neri AL, Pinto MEB, Sommerhalder C, Perracini MR, Yuaso DR. Cuidar de idosos no contexto da família: questões psicológicas e sociais. Campinas (SP): Alínea; 2002. p.165-201.

4 Santos SMA Idosos, família e cultura: um estudo sobre a construção do papel do cuidador. Campinas, S P: Alínea; 2003.

5 Alvarez AM, Gonçalves LHT. Tendo que cuidar: a vivência do idoso e da família cuidadora no processo de cuidar e ser cuidado em contexto domiciliar. Texto Contexto Enferm. 2001 Maio-Ago; 10 (2): 205-7.

6 Mazza MMPR, Lefevre F. Cuidar em família: análise da representação social da relação do cuidador familiar com o idoso. Rev. Bras. Crescimento Desenvolv. Humano. 2005 Jan-Jun; 15 (1): 1-10.

7 Garrido R, Menezes PR. Impacto em cuidadores de idosos com demência atendidos em um serviço psicogeriátrico. Rev. Saúde Públ. 2004 Nov-Dez; 38 (6): 835-41.

8 Meira EC, Gonçalves LHT, Silva JAS, Souza AS, Neri IG. Fatores de risco de maus tratos ao idoso na relação idoso/cuidador em convivência intrafamiliar. Textos Envelhecimento. 2004 Jul-Dez; 7 (2): 63-84.

9 Fleck MPA, coordenador. Versão em português do instrumento de avaliação de Qualidade de Vida (WHOQOL) 1998: WHOQOL abreviado-versão em português [acesso 2006 fev 27]. Disponível em: www. ufrgs.br/psiq/whoqol84.html

10 Grupo de Estudos sobre cuidado de Saúde de Pessoas Idosas (GESPI). Diretório dos Grupos de Pesquisa no Brasil [acesso em 2006 fev 28]. Disponível em: http: //dpg.cnpq.br/buscanacional/

11 Ministério de Trabajo y Asuntos Sociales (ES). Cuidados en la vejez: el apoyo informal. 2a reimp. Madrid: IMSERSO; 1999.

12 Budó MLD. A mulher como cuidadora no contexto de uma comunidade rural de imigração italiana. Texto Contexto Enferm. 1997 Jan-Abr; 6 (1): 181-97.

13 Sommerhalder C. Significados associados a tarefa de cuidar de idosos de alta dependência no contexto familia [dissertação]. Campinas (SP): UNICAMP/ Faculdade de Educação; 2001.

14 Meleau-Ponty M. Fenomenologia da percepção. 2a ed. São Paulo (SP): Martins Fontes; 1999.

15 Boff L. Cuidar da vida e da criação. In: Beozzo JO, organizador. Saúde: cuidar da vida e da integridade da criação. São Paulo (SP): CESEP; 2002. p.89-108. 